\title{
Prolonged left ventricular dysfunction following pharmacologic stress myocardial perfusion imaging: Should we be less stunned than in the past?
}

\author{
Andrew Van Tosh, MD, a and Kenneth J. Nichols, PhD ${ }^{\mathrm{b}, \mathrm{c}}$ \\ a Noninvasive Imaging Laboratory, From the Research Department, St. Francis Hospital, Roslyn, \\ NY \\ b Department of Radiology, Donald and Barbra Zucker School of Medicine at Hofstra/Northwell, \\ Hempstead, NY \\ c Division of Nuclear Medicine and Molecular Imaging, Northwell Health, New Hyde Park, NY
}

Received Jul 23, 2018; accepted Jul 23, 2018

doi: $10.1007 / \mathrm{s} 12350-018-1389-2$

\section{See related article, https://doi.org/10.10 07/s1 2350-018-1340-6.}

SPECT myocardial perfusion imaging (MPI) with ECG-gated equilibrium wall motion analysis is the most utilized non-invasive tool for the diagnosis and management of coronary disease. It provides precise localization of coronary stenoses, global left ventricular (LV) function, regional wall motion, and asynchrony. Recent analyses indicate that sensitivities for detecting coronary disease are in the range of $76 \%$ to $81 \%$, with specificities as high as $71 \% .^{1}$ MPI also imparts significant prognostic information. The extent of perfusion defects and their degree of reversibility correlate with adverse coronary events including MI and cardiac death. $^{2}$ This applies to MPI performed either with exercise or pharmacologic stress.

One limitation of SPECT MPI has been that its sensitivity for identifying multi-vessel coronary disease (MVD) may be $<50 \%$. $^{3-5}$ MVD strongly affects CAD prognosis and, when accompanied by LV dysfunction, is a primary indication for revascularization. This finding has been attributed to several factors. ${ }^{6}$ The spatial res-

Reprint requests: Andrew Van Tosh, MD, Noninvasive Imaging Laboratory, From the Research Department, St. Francis Hospital, 100 Port Washington Blvd., Roslyn, NY 11576-1348; Andrew.VanTosh@chsli.org

J Nucl Cardiol 2021;28:718-22.

$1071-3581 / \$ 34.00$

Copyright (C) 2018 American Society of Nuclear Cardiology. olution of SPECT may limit its ability to separate perfusion defects in neighboring territories, particularly in small hearts. ${ }^{7}$ Certain perfusion defects, particularly of the left main coronary artery, are not well detected on SPECT MPI, limiting recognition of MVD affecting the left main. ${ }^{8}$ Flow-dependent extraction fraction of radionuclides may blunt regional differences in tracer uptake at high cardiac outputs achieved in vasodilator stress. Finally, qualitative or semi-quantitative diagnosis of coronary disease requires comparison of relative hypo-perfusion in one territory to a region which appears normally perfused. In patients with MVD, regions with "normal" perfusion may still be supplied by stenotic arteries, resulting in under-diagnosis of CAD extent. ${ }^{6}$

Adjunctive scintigraphic parameters have been used to improve the identification of MVD on perfusion imaging. Lung tracer uptake, first quantified as a lungto-heart ratio on anterior planar Tl-201 images, was shown to correlate with LV dysfunction and extent of CAD. The association of increased lung tracer uptake, increased LV filling pressure, and MVD remains valid for SPECT with Tc-99m tracers. Transient ischemic dilation of the LV cavity (TID), caused by LV enlargement, subendocardial ischemia, or systolic dysfunction, was initially quantified as the area of the stress/ delayed images on planar Tl-201 studies. ${ }^{9}$ TID achieved a $60 \%$ sensitivity for MVD (ratio 1.17 ), ${ }^{10}$ improving to $70 \%$ using an automated method on dual-isotope SPECT studies. TID also predicts $\mathrm{MACE}^{11}$ and remains an important prognostic parameter.

Braunwald first defined the concept of stunning in 1982 as a myocardial injury which may persist for up to 
several days following an ischemic insult, ${ }^{12}$ and caused by abnormal myocardial calcium accumulation or liberation of oxidizing free radicals. ${ }^{13}$ Myocardial stunning on SPECT MPI is evidenced by a decrease in LV ejection fraction (LVEF) from rest to post-stress images of $\geq 5 \%$, based on the variability of gated LVEF measurements in patients studied at rest on two successive days. ${ }^{14}$ The most important determinant of myocardial stunning is the extent of CAD. Hida et al. found that myocardial stunning had a sensitivity of $66 \%$ for severe $\mathrm{CAD},{ }^{2}$ while Druz et al. reported that coronary jeopardy scores directly correlate with myocardial stunning. ${ }^{15}$ Its prevalence varies from $3 \%$ to $36 \%$, also dependent on CAD severity. ${ }^{16}$ The time course of stunning has not been established. When image acquisition is simultaneous with the administration of stress agent, as in PET, a decrease in LVEF vs rest may be seen immediately, and correlates with severely reduced perfusion and coronary steal. ${ }^{17,18}$ When image acquisition occurs in the post-stress time period (SPECT), reduced LVEF can be documented in ischemic patients at 4 minutes of recovery, becoming less pronounced at nine minutes through 21 minutes. The prevalence of myocardial stunning decreases from 15 to 60 minutes of recovery. ${ }^{19}$ Ischemic LV dysfunction begins early after stress, with a variable time course. However, the precise time intervals for assessing myocardial stunning post stress have not been defined.

The presence of "reversible" regional wall motion abnormalities (RWMA), present on post-stress but not rest imaging, has also been used to identify severe CAD or MVD. Some patients decrease LVEF from rest to stress, but do not meet the $5 \%$ criterion established by Johnson. ${ }^{14}$ Worsening RWMA determined qualitatively was specific for CAD and had a $90 \%$ positive predictive value for severe stenoses and three vessel CAD., ${ }^{40}$ Reversible RWMA is prognostically important, and was the strongest predictor of MACE in patients without MI, in the study by Petix et al. ${ }^{21}$ RWMA may be a more sensitive predictor than a $\triangle \mathrm{LVEF}$ of $5 \%$ for identifying severe CAD or predicting cardiac events.

Included in the approaches to assessing RWMA by scintigraphic imaging is the quantification of LV wall thickening (WT). The physical principles underlying the use of LV WT, and the change in segmental WT from rest to stress, were first described by Hoffman. Using PET data, ${ }^{22}$ he noted that the partial volume effectdecreased counts for structures smaller than twice the spatial resolution of an imaging device-can be used to measure myocardial wall thickening by way of increasing myocardial counts from diastole to systole. As demonstrated by Galt et al., ${ }^{23}$ the typical $1-\mathrm{cm}$ transaxial spatial resolution of SPECT imaging lends itself well to measure myocardial wall thickening, as
CMRI data demonstrate that the myocardial wall thickness is typically $1 \mathrm{~cm}$ at diastole, ${ }^{24}$ so that the change in maximum regional myocardial counts should be adequately modeled as a linear relationship to the change in myocardial wall thickness. ${ }^{25,26}$ Wall thickening may be measured qualitatively, or by using automated quantified algorithms. Visual wall thickening analysis was used by Bestetti et al. to evaluate 138 patients with CAD. Wall thickening scores were significantly higher (more abnormal), in patients with more severe angiographic disease. ${ }^{27}$ Automated algorithms to evaluate wall thickening were described by Germano et al. who compared a wall thickening algorithm derived from phantoms with qualitative visual assessment of wall 'brightening', in 79 patients with varying LVEF. Concordance was $90 \% .^{28}$ Validity of these assumptions and the limits of precision with which WT can be measured by gated SPECT have been validated directly against cardiac MRI. ${ }^{29}$ The QGS algorithms measure wall thickening by tracking the endocardial and epicardial borders encompassing the myocardial counts, while incorporating count increases via the partial volume effect with shape tracking, and constraining the overall computed myocardial mass to be constant throughout all segments of the R-R interval used to acquire the gated SPECT data. ${ }^{30}$ Thus, regional WT values are among the many parameters that are quantifiable by gated SPECT, and additional features can be derived.

In the current issue of the Journal, Bestetti et al. evaluate the utility of using change in wall thickening from rest to stress as a marker of myocardial stunning and severe CAD in 52 patients undergoing dipyridamole Tc-99m stress MPI using a 2-day protocol. All patients had inducible ischemia (SDS $>5$ ) and coronary angiography within three months showing at least one major vessel with $\geq 70 \%$ stenosis. QGS/QPS algorithms were used to generate standard relative perfusion scores, wall motion, LV volume, and LVEF. Automated wall thickening SSS, SRS, and SDS were generated. The principle results were that $60 \%$ of patients had MVD, and LVEF decreased 2.4 units, and WT-SDS increased (worsened) by 3.97. Patients with severe ischemia (SDS $\geq 8$ ) had significantly higher WT-SDS than those with moderate ischemia (SDS 5 to 7), but $\triangle \mathrm{LVEF}$ did not differ. Regression analysis showed a moderate correlation between WT-SDS and perfusion SDS and $\triangle \mathrm{LVEF}$.

The study is important in its attempt to establish the parameter of wall thickening (rest vs stress) as an analogue for myocardial stunning. In their hands WT-SDS appears to be a more sensitive marker of CAD than $\triangle \mathrm{LVEF}$, and changes significantly even when $\triangle \mathrm{LVEF}$ remains static. Among the positive aspects of this study are that there were angiographic data establishing the 
extent of CAD, and myocardial perfusion SDS and WTSDS values were obtained quantitatively by automated algorithms,${ }^{31}$ rather than subjectively.

The changes in wall thickening noted in the study were significant, but modest: 3.97 for the patient cohort as a whole, and 5.97 for patients with severe ischemia. In the study of $\mathrm{Xu}$ et al., the variability of wall thickening scores was 5\%, within the same order of magnitude of as the difference in WT in moderate vs severe ischemic patients in the present study. ${ }^{32}$

This limitation in measurement precision contributes to the difficulty in appreciating the importance of the results, but there are additional factors as well. First, the number of patients in the two subgroups, moderate vs severe ischemia, is not given. Whether there was sufficient sample size to be reliable or meaningful is uncertain, a problem that could have been addressed with power analysis.

Second, the study fails to establish the authors' own variability of the WT parameter, such as was performed in the study of Johnson et al. ${ }^{14}$ This could have been accomplished by reporting the results of WT-SDS in a control group without ischemia or with mild ischemia. Alternatively, a test/re-test protocol could have been carried out in a small subgroup of the cohort. Such analyses would have provided a gauge of the magnitude of noise in the data and an indication of the limits of WT-SDS measurement precision. Also, in comparing WT-SDS among groups, the paired $t$ test was applied, but this would only have been valid if the distribution was normally distributed. Otherwise, the Mann-Whitney test should have been applied, and the significance of the results might have been affected.

Third, the study leaves unanswered the question of what wall thickening criteria are to be used to define myocardial stunning. In part, this is due to the lack of information on measurement reproducibility, as noted above. However, the authors used a regression equation (Figure 1A) to indicate that a WT-SDS score of 10 was required to cause a $\triangle \mathrm{LVEF}$ of $-5 \%$, suggesting that these parameters be considered equivalent. Inspection of the graph data, though, reveals at least 6 to 7 patients with WT-SDS $<10$ had $\triangle \mathrm{LVEF}$ decrease of more than $5 \%$, invalidating this concept. The authors do not relate WT-SDS to coronary anatomy, presumably because of the small numbers involved, but this would have been another mechanism with which to separate limits of normal and abnormal.

When myocardial stunning was originally described on gated SPECT data by Johnson, et al, ${ }^{14}$ a prevalence of $36 \%$ of patients was reported. However, over the last two decades, changes in practice patterns and referral bias have led to a reduction in the percentage of abnormal and severely abnormal SPECT MPI studies seen in most laboratories, so the expected prevalence of stunning would now be less. The current paper does not specifically detail how many patients would fulfill the $\triangle \mathrm{LVEF}$ decrease of $5 \%$. Estimating from Figure $1 \mathrm{~A}$, the number is approximately 13 to 14 , for a prevalence of 25 to $27 \%$, less than previously, but within a similar range. Requiring the subjects to have a perfusion $\operatorname{SDS} \geq 5$ has resulted in a higher risk group than most laboratories' current referral populations. The true prevalence of stunning in a laboratory population typical of 2018, whether defined by $\triangle \mathrm{LVEF}$ or WT-SDS, will need further study in a non-selected group.

The pathophysiology of patients with myocardial stunning, particularly following vasodilator stress, may be further characterized using rest-stress PET perfusion imaging. The gated list mode stress data for an ${ }^{82} \mathrm{Rb}$ PET protocol are acquired, in their entirety, simultaneous with peak pharmacologic stress. Doing so has shown that global and regional LV function changes during stress can be related to abnormal global and regional myocardial blood flow, flow reserve, and coronary resistance. ${ }^{33}$ Changes in LV asynchrony, quantified as the phase of regional wall contraction, correspond to quantified arteriographic findings. ${ }^{34}$

\section{FUTURE DIRECTIONS}

The advent of newer solid-state detectors that acquire data from $180^{\circ}$ simultaneously, and that have count sensitivities 3 to 5 greater than those of conventional Anger detectors, ${ }^{35}$ enables continuous data collection over 30 to 60 minutes, which could then be re-binned into sequential time segments during and after pharmacologic stress. By these means, the time course of change in LV function and myocardial stunning may be defined in greater detail, documenting the sequence by which a patient's ventricular function transforms back to a resting state. The study of Brodov et al. examined more precisely the time sequence of ischemic myocardial dysfunction using solid-state technology. ${ }^{36}$ Fifty patients underwent regadenoson rest/stress MPI. Two-minute post-stress acquisitions were performed at four minute intervals beginning one minute after stressor and isotope injection. Patients with significant ischemia had a drop in LVEF of $4.6 \%$ at 5 through 9 minutes, gradually returning toward normal at 21 minutes. Those with mild or no ischemia had increased LVEF. This pilot study was the first to examine in this degree of detail the time course of post-stress LV functional changes. ${ }^{36}$ Further studies need to be performed to evaluate relationships to coronary anatomy, and to regional function and asynchrony, if adequate signal-to-noise ratios can be obtained. Such data would provide a clearer picture of the prevalence and time course of 
transient myocardial stunning, potentially enabling more refined risk stratification of patients.

\section{Disclosure}

Andrew Van Tosh serves as a consultant to Astellas Pharma Global Development, Inc; Kenneth Nichols participates in royalties from Syntermed, Inc.

\section{References}

1. McArdle BA, Dowsley TF, deKemp RA, Wells GA, Beanlands RS. Does Rubidium-82 PET have superior accuracy to SPECT perfusion imaging for the diagnosis of obstructive coronary disease?: A systematic review and meta-analysis. J Am Coll Cardiol 2012;60:1828-37.

2. Hida S, Chikamori T, Tanaka H, Usui Y, Igarashi Y, Nagao T, et al. Diagnostic value of left ventricular function after stress and at rest in the detection of multivessel coronary artery disease as assessed by electrocardiogram-gated SPECT. J Nucl Cardiol 2007; 14:68-74.

3. Bateman TM, Heller GV, McGhie AI, Friedman JD, Case JA, Bryngelson JR, et al. Diagnostic accuracy of rest/stress ECG-gated Rb-82 myocardial perfusion PET: Comparison with ECG-gated Tc-99m sestamibi SPECT. J Nucl Cardiol 2006;13:24-33.

4. Shirai N, Yamagishi H, Yoshiyama M, Teragaki M, Akioka K, Takeuchi K, et al. Incremental value of assessment of regional wall motion for detection of multivessel coronary artery disease in exercise 201tl gated myocardial perfusion imaging. J Nucl Med 2002;43:443-50

5. Hida S, Chikamori T, Tanaka H, Igarashi $\mathrm{Y}$, Hatano T, Usui $\mathrm{Y}$, et al. Diagnostic value of left ventricular function after adenosine triphosphate loading and at rest in the detection of multi-vessel coronary artery disease using myocardial perfusion imaging. $\mathrm{J}$ Nucl Cardiol 2009;16:20-7.

6. Travin MI. Is it possible for myocardial perfusion imaging to avoid missing any patients with high-risk coronary disease? J Nucl Cardiol 2007; 14:492-5.

7. Hansen CL, Crabbe D, Rubin S. Lower diagnostic accuracy of thallium-201 SPECT myocardial perfusion imaging in women: An effect of smaller chamber size. J Am Coll Cardiol 1996;28:1214-9.

8. Berman DS, Kang X, Slomka PJ, Gerlach J, de Yang L, Hayes SW. Underestimation of extent of ischemia by gated SPECT myocardial perfusion imaging in patients with left main coronary artery disease. J Nucl Cardiol 2007;14:521-8.

9. McLaughlin MG, Danias PG. Transient ischemic dilation: A powerful diagnostic and prognostic finding of stress myocardial perfusion imaging. J Nucl Cardiol 2002;9:663-7.

10. Weiss AT, Berman DS, Lew AA, Nielsen J, Potkin B, Swan HJC, et al. Transient ischemic dilation of the left ventricle on stress Thallium-201 scintigraphy: A marker of severe and extensive coronary artery disease. J Am Coll Cardiol 1987;9:752-9.

11. Bestetti A, Bigi R, Terranova P, Lombardi F, Fiorentini C. Prognostic implications of stress-induced transient ischemic dilation of the left ventricle in patients with systolic dysfunction and fixed perfusion defects. Int J Cardiol 2010;140:323-7.

12. Braunwald E, Kloner RA. The stunned myocardium: Prolonged, postischemic ventricular dysfunction. Circulation 1982;66:1146-9.

13. Bolli R. Mechanism of myocardial "stunning.". Circulation 1990;82:723-38
14. Johnson LL, Verdesca SA, Aude WY, Xavier RC, Nott LT, Campanella MN, et al. Postischemic stunning can affect left ventricular ejection fraction and regional wall motion on poststress gated Sestamibi tomograms. J Am Coll Cardiol 1997;30:1641-8.

15. Druz RS, Akinboboye OA, Grimson R, Nichols KJ, Reichek N. Postischemic stunning after adenosine vasodilator stress. J Nucl Cardiol 2004;11:534-41.

16. Ramakrishna G, Miller TD, Hodge DO, O'Connor MK, Gibbons RJ. Differences in left ventricular ejection fraction and volumes measured at rest and poststress by gated sestamibi SPECT. J Nucl Cardiol 2006;13:668-74.

17. Dorbala S, Vangala D, Sampson U, Limaye A, Kwong R, Di Carli MF. Value of vasodilator left ventricular ejection fraction reserve in evaluating the magnitude of myocardium at risk and the extent of angiographic coronary artery disease: A $82 \mathrm{Rb}$ PET/CT study. J Nucl Med 2007;48:349-58.

18. Van Tosh A, Votaw JR, Reichek N, Palestro CJ, Nichols KJ. The relationship between ischemia-induced left ventricular dysfunction, coronary flow reserve and coronary steal on regadenoson stress gated ${ }^{82} \mathrm{Rb}$ PET myocardial perfusion imaging. J Nucl Cardiol 2013;20:1060-8.

19. Mut F, Giubbini R, Vitola J, Lusa L, Sobic-Saranovic D, Peix A, et al. Detection of post-exercise stunning by early gated SPECT myocardial perfusion imaging: Results from the IAEA multicenter study. J Nucl Cardiol 2014;21:1168-76.

20. Emmett L, Iwanochko RM, Freeman MR, Barolet A, Lee DS, Husain M. Reversible regional wall motion abnormalities on exercise technetium-99m gated cardiac single photon emission computed tomography predict highgrade angiographic stenoses. J Am Coll Cardiol 2002;39:991-8.

21. Petix NR, Sestini S, Marcucci G, Coppola A, Arena A, Nassi F, et al. Can the reversible regional wall motion abnormalities on stress gated Tc-99m sestamibi SPECT predict a future cardiac event? J Nucl Cardiol 2005;12:20-31.

22. Hoffman EJ, Huang SC, Phelps ME. Quantitation in positron emission computed tomography: I. Effects of object size. J Comput Assist Tomogr 1979;5:391-400.

23. Galt JR, Garcia EV, Robbins WL. Effects of myocardial wall thickness of SPECT quantification. IEEE Trans Med Imaging 1990;9:144-50.

24. Pflugfelder PW, Sechtem UP, White RD, Higgins CB. Quantification of regional myocardial function by rapid cine MR imaging. AJR 1988;150:523-9.

25. Faber TL, Akers MS, Peshock RM, Corbett JR. Three-dimensional motion and perfusion quantification in gated single-photon emission computed tomograms. J Nucl Med 1991;32:2311-7.

26. Faber TL, Cooke DC, Folks RD, Vansant JP, Nichols KJ, DePuey $\mathrm{EG}$, et al. Left ventricular function from gated SPECT perfusion images: An integrated method. J Nucl Med 1999;40:650-9.

27. Bestetti A, Di Leo C, Alessi A, Triulzi A, Tagliabue L, Tarolo GI. Post-stress end-systolic left ventricular dilation: A marker of endocardial post-ischemic stunning. Nucl Med Commun 2001;22:685-93.

28. Germano G, Erel J, Lewin H, Kavanagh PB, Daniel S, Berman DS. Automatic quantitation of regional myocardial wall motion and thickening from gated technetium- $99 \mathrm{~m}$ sestamibi myocardial perfusion single-photon emission computed tomography. J Am Coll Cardiol 1997;30:1360-7.

29. Nichols KJ, Van Tosh A, Wang Y, Faber TL, Palestro CJ, Reichek N. Correspondence between gated SPECT and cardiac magnetic resonance quantified myocardial wall thickening. Int J Cardiovasc Imaging 2011;27:1095-104. 
30. Germano G, Kiat H, Kavanaugh PB, et al. Automatic quantification of ejection fraction from gated myocardial perfusion SPECT. J Nucl Med 1995;36:2138-47.

31. Slomka PJ, Berman DS, Xu Y, Kavanagh P, Hayes SW, Dorbala S, et al. Fully automated wall motion and thickening scoring system for myocardial perfusion SPECT: Method development and validation in large population. J Nucl Cardiol 2012;19:291-302.

32. Xu Y, Hayes S, Ali I, Ruddy TD, Wells RG, Berman DS, et al. Automatic and visual reproducibility of perfusion and function measures for myocardial perfusion SPECT. J Nucl Cardiol 2010;17:1050-7.

33. Van Tosh A, Votaw JR, Cooke CD, Reichek N, Palestro CJ, Nichols KJ. Relationships between left ventricular asynchrony and myocardial blood flow. J Nucl Cardiol 2017;24:43-52.
34. Van Tosh A, Votaw JR, Cooke CD, Cao J, Palestro CJ, Nichols KJ. Relationship of ${ }^{82} \mathrm{Rb}$ PET territorial myocardial asynchrony to arterial stenosis. J Nucl Cardiol 2018. https://doi.org/10.1007/s12 350-018-1350-4.

35. Slomka PJ, Patton JA, Berman DS, Germano G. Advances in technical aspects of myocardial perfusion SPECT imaging. J Nucl Cardiol 2009; 16:255-76.

36. Brodov Y, Fish M, Rubeaux M, Otaki Y, Gransar H, Lemley M, et al. Quantitation of left ventricular ejection fraction reserve from early gated regadenoson stress Tc-99m high-efficiency SPECT. J Nucl Cardiol 2016;23:1251-61. 